\title{
Numerical integration scheme for singular integrals based on polar coordinates free from angular quasi-singularities
}

\author{
J.D.R. Bordón, J.J. Aznárez, O. Maeso \\ Instituto Universitario de Sistemas Inteligentes y Aplicaciones Numéricas en Ingeniería, \\ Universidad de Las Palmas de Gran Canaria, Edificio Central del Parque Científico y Tecnológico del \\ Campus Universitario de Tafira, 35017 Las Palmas de Gran Canaria, Spain \\ \{jacobdavid.rodriguezbordon, juanjose. aznarez, orlando.maeso\}@ulpgc.es \\ Pre-print of the paper originally published in Engineering Analysis with Boundary Elements, 103:126-136, 2019. \\ DOI: http://dx.doi.org/10.1016/j.enganabound.2019.03.010 Journal version available under May 9, 2019 via \\ https://authors.elsevier.com/a/1YlLh3PKjshw6X
}

\begin{abstract}
Boundary element method formulations usually rely eventually on the calculation of weakly singular integrals, and hence robust and efficient algorithms for their evaluation are desirable. This paper proposes a numerical scheme based on a conformal polar transformation, four novel non-linear angular transformations, and a subdivision pattern which allows treating both triangular and quadrilateral elements in a common framework. It is shown that this scheme has small sensitivity to the location of the collocation point and the element aspect ratio and skewness because all sources of angular quasi-singularities have been removed by the non-linear transformations. The proposed methodology is compared against others where its robustness and efficacy is demonstrated.

Keywords: numerical integration, polar coordinates, singular integrals, boundary element method
\end{abstract}

\section{Introduction}

The Boundary Element Method (BEM) is nowadays used in many areas of the industry where its advantages are exploited. The effort of numerous researchers and developers has allowed the BEM to be a well established methodology [5, 3, 2]. One of the key issues is the correct and efficient evaluation of the integrals arising in the method, which require the use of specific strategies.

Integrals should preferably be evaluated analytically, but this can only be done in certain cases, usually for planar and low-order elements, and simple fundamental solutions. However, boundary element integrals are generally evaluated by numerical integration due to its versatility. Before performing the numerical integration, some analytical transformation (subdivision, change of variables, integration by parts) may be applied in order facilitate the computation, or even being able to evaluate the integral at all. The number of developed mathematical and computational techniques for handling boundary element integrals is quite large, see e.g. [20], and these focus mainly on two of the most problematic ones: nearly (or quasi) singular integrals and singular integrals, which can contain a weak, strong or hyper singularity. Strongly singular and hypersingular integrals can be reduced to weakly singular integrals, other non-singular (possibly nearly singular) integrals and analytical terms via regularization procedures like the use of simple solutions (regularization before discretization) [4, 6, 1] or the use of subtraction and addition of integrand expansions via Guiggiani's method (regularization after discretization) [8, 9, 12, 7].

For weakly singular integrals over surface elements, many approaches

${ }^{*}$ Corresponding author have been devised, from which the classical quadrilateral-to-triangle degenerated mapping [14] and the use of polar coordinates properly deals with the problem. In both cases, the element is subdivided into triangular regions with vertices at the element vertices and at the collocation point. They suffer from two serious defects which affect their efficiency: dependency on the location of the collocation point and element shape (aspect ratio and skewness). The first defect arises from strong quasisingularities in the angular coordinate, and it can be treated with classical techniques such as $h$ - and/or $p$ - refinement [18] or non-linear transformations [13, 19]. The second defect also also arises from quasisingularities appearing when tangent vectors along local coordinates are neither equal nor orthogonal at the collocation point, i.e. not conformal. Rong et al. [19] proposed a conformal polar transformation which imposes exactly this condition for triangular elements. They used sigmoidal transformations from Johnston [11] in order to treat the strong quasi-singularities. However, there is a previous exact transformation proposed by Khayat et al. [13] which completely cancels out these. Rong et al. [19] reported that their experience showed that Khayat's transformation generally is worse than sigmoidal transformations, but there was no clear reasoning why this was happening. Therefore, there is a theoretical gap between both works which we aim to fill.

In the present work, Rong's conformal polar transformation is generalized for quadrilateral elements in a different fashion to Lv et al. [15]. Also, four novel non-linear transformations which completely removes the strong angular quasi-singularities are proposed and theoretically and numerically studied. These ingredients are put together to make a robust and efficient numerical scheme.

The rest of the paper is organized as follows. In Section 2 the proposed numerical scheme is described in detail. Section 3 presents and compares the novel and previous non-linear transformations for treating the strong angular quasi-singularities. In Section 4 the methodology is used in a number of examples for both triangular and quadrilateral elements. Finally, conclusions are given in Section 5

\section{Numerical integration scheme}

The numerical integration scheme is based on polar coordinates transformation with a set of additional transformations which makes it robust, efficient and applicable to both triangular and quadrilateral elements. The presence of two known sources of angular quasi-singularities deteriorate the performance of basic polar coordinates transformations. These two sources of quasi-singularities are:

Primary Angular quasi-singularities appearing when the collocation point approaches the element edges. This is due to the severe 
change of the distance between the collocation point and the element edge when the collocation point is close the element edge.

Secondary Angular quasi-singularities appearing on elements with high aspect ratios and skewness. This is due to the distortion of polar coordinates mapping between the reference space and the real space in the limit $r \rightarrow 0$ when using conventional polar coordinates transformations.

A graphical summary of the proposed numerical scheme is depicted in Fig. 11 It consists of the following six transformations described stepby-step as follows:

1. Transformation from the element reference space to the real space $T_{\mathrm{g}}: \xi \rightarrow \mathbf{x}:$

$$
\mathbf{x}(\xi)=\sum_{k=1}^{N_{\mathrm{n}}} \phi^{(k)}(\xi) \cdot \mathbf{x}^{(k)}
$$

where $N_{\mathrm{n}}$ is the number of nodes of the geometric interpolation, and $\phi^{(k)}(\xi)$ and $\mathbf{x}^{(k)}$ are respectively the shape function and position vector of node $k$. The Jacobian $J_{\mathrm{g}}$ of the transformation of areas $\left(\mathrm{d} \Gamma=J_{\mathrm{g}} \mathrm{d} \xi_{1} \mathrm{~d} \xi_{2}\right)$ is the usual:

$$
J_{\mathrm{g}}=\left|\frac{\partial \mathbf{x}}{\partial \xi_{1}} \times \frac{\partial \mathbf{x}}{\partial \xi_{2}}\right|=\left|\mathbf{T}_{1}^{\xi} \times \mathbf{T}_{2}^{\xi}\right|=\left|\mathbf{N}^{\xi}\right|
$$

where $\mathbf{T}_{1}^{\xi}$ and $\mathbf{T}_{2}^{\xi}$ are the tangent vectors of curvilinear coordinates $\xi_{1}$ and $\xi_{2}$ respectively, and $\mathbf{N}^{\xi}$ is the corresponding normal vector of the tangent plane.

2. Transformation from the subdivision reference space to the subdivision $\mathrm{S} d$ on the element reference space $T_{\mathrm{S} d}: \zeta \rightarrow \xi$ :

$$
\begin{aligned}
& \xi=\xi^{\mathrm{i}}+\mathbf{S}^{\mathrm{S} d} \zeta \\
& \mathbf{S}^{\mathrm{S} d}=\left(\begin{array}{ll}
\xi_{1}^{(1)-S d}-\xi_{1}^{\mathrm{i}} & \xi_{1}^{(2)-\mathrm{S} d}-\xi_{1}^{\mathrm{i}} \\
\xi_{2}^{(1)-S d}-\xi_{2}^{\mathrm{i}} & \xi_{2}^{(2)-\mathrm{S} d}-\xi_{2}^{\mathrm{i}}
\end{array}\right)
\end{aligned}
$$

where the Jacobian is $J_{\mathrm{S} d}=\left|\partial\left(\xi_{1}, \xi_{2}\right) / \partial\left(\zeta_{1}, \zeta_{2}\right)\right|=\operatorname{det}\left(\mathbf{S}^{\mathrm{S} d}\right)$. The element is subdivided at this transformation into $N_{\mathrm{S}}$ subdivisions, i.e. $d=1, \ldots, N_{\mathrm{S}}$. Therefore, from this point on, transformations are subdivision-dependent and denoted using superscript $\mathrm{S} d$. In order to deal with arbitrary locations of the collocation point, the element subdivision pattern is based on splitting the element into triangles using the collocation point as the common vertex, and the other vertices at the element vertices. Fig. 2 shows this classical subdivision pattern, where each triangular subdivision are denoted as $\mathrm{S} d$. In the general case, there are as many triangles as element edges, except when the collocation point is located at an edge or a vertex, in which cases respectively one or two of the triangles degenerate and does not contribute to the integral.

3. Transformation from the conformal subdivision space to the subdivision reference space $T_{\mathrm{C} d}: \eta \rightarrow \zeta$ :

$$
\begin{aligned}
& \zeta=\mathbf{C}^{\mathrm{S} d}\left(\eta-\eta^{\mathrm{i}-\mathrm{S} d}\right) \\
& \mathbf{C}^{\mathrm{S} d}=\left(\begin{array}{cc}
-1 & \left(\eta_{1}^{\mathrm{i}-\mathrm{S} d}-1\right) / \eta_{2}^{\mathrm{i}-\mathrm{S} d} \\
1 & -\eta_{1}^{\mathrm{i}-\mathrm{S} d} / \eta_{2}^{\mathrm{i}-\mathrm{S} d}
\end{array}\right)
\end{aligned}
$$

where the Jacobian $J_{\mathrm{C} d}=\left|\partial\left(\zeta_{1}, \zeta_{2}\right) / \partial\left(\eta_{1}, \eta_{2}\right)\right|$ is simply:

$$
J_{\mathrm{C} d}=1 / \eta_{2}^{\mathrm{i}-\mathrm{S} d}
$$

The purpose of this transformation is to suppress the secondary quasi-singularities. The idea is to provide an advantageous space where polar coordinates have a conformal map to the real space. To the authors' best knowledge, this idea can be traced back to the work of Hayami et al. [10] (PART method) for quasi-singular integrals, who introduced the polar coordinates in a projection of the element onto a plane with normal coincident with the normal of the element at the point nearest to the collocation point. In this way, the mapping of polar coordinates to the real space is very good throughout the element, i.e. there is an approximate proportionality between $\rho$ and $r$ with very small dependence on the angular coordinate. More recently, Rong et al. [19] used the same idea for singular integrals, but only at collocation points where this mapping goodness is imposed through the exact fulfilment of conformality conditions. Rong et al. [19] developed this methodology for triangular elements, while Lv et al. [15] did the same for quadrilateral elements. The present scheme however allows the treatment of both types of elements.

The location of the collocation point in the $\eta$ space, i.e. $\eta^{\mathrm{i}-\mathrm{S} d}$, is such that conformality of polar coordinates is present at the collocation point in the real space. For each subdivision $d, \eta^{\mathrm{i}-\mathrm{S} d}$ must fulfill the following conformality conditions at the collocation point:

I Equal length:

$$
\left(\mathbf{T}_{1}^{\eta}\right)^{\mathrm{i}} \cdot\left(\mathbf{T}_{1}^{\eta}\right)^{\mathrm{i}}=\left(\mathbf{T}_{2}^{\eta}\right)^{\mathrm{i}} \cdot\left(\mathbf{T}_{2}^{\eta}\right)^{\mathrm{i}}
$$

II Orthogonality:

$$
\left(\mathbf{T}_{1}^{\eta}\right)^{\mathrm{i}} \cdot\left(\mathbf{T}_{2}^{\eta}\right)^{\mathrm{i}}=0
$$

where:

$$
\left(\mathbf{T}_{j}^{\eta}\right)^{\mathrm{i}}=\left(\frac{\partial \mathbf{x}}{\partial \eta_{j}}\right)^{\mathrm{i}}=\left(\frac{\partial \mathbf{x}}{\partial \xi_{l}}\right)^{\mathrm{i}} \frac{\partial \xi_{l}}{\partial \zeta_{k}} \frac{\partial \zeta_{k}}{\partial \eta_{j}}=\mathbf{T}_{l}^{\xi} S_{l k}^{\mathrm{Sd}} C_{k j}^{\mathrm{Sd}}
$$

where $j, k, l=1,2$ and Einstein summation convention is implied. The solution can be written as:

$$
\begin{aligned}
& \eta_{1}^{\mathrm{i}-\mathrm{S} d}=-\frac{a_{l} b_{k}\left[\left(\mathbf{T}_{l}^{\xi}\right)^{\mathrm{i}} \cdot\left(\mathbf{T}_{k}^{\xi}\right)^{\mathrm{i}}\right]}{a_{l} a_{k}\left[\left(\mathbf{T}_{l}^{\xi}\right)^{\mathrm{i}} \cdot\left(\mathbf{T}_{k}^{\xi}\right)^{\mathrm{i}}\right]} \\
& \eta_{2}^{\mathrm{i}-\mathrm{S} d}=\sqrt{\frac{b_{l} b_{k}\left[\left(\mathbf{T}_{l}^{\xi}\right)^{\mathrm{i}} \cdot\left(\mathbf{T}_{k}^{\xi}\right)^{\mathrm{i}}\right]}{a_{l} a_{k}\left[\left(\mathbf{T}_{l}^{\xi}\right)^{\mathrm{i}} \cdot\left(\mathbf{T}_{k}^{\xi}\right)^{\mathrm{i}}\right]}-\left(\eta_{1}^{\mathrm{i}-\mathrm{S} d}\right)^{2}}
\end{aligned}
$$

where $a_{j}=\xi_{j}^{(2)-\mathrm{S} d}-\xi_{j}^{(1)-\mathrm{S} d}$ and $b_{j}=\xi_{j}^{(1)-\mathrm{S} d}-\xi_{j}^{\mathrm{i}}$, and the notation is such that summation convention expands independently for numerator and denominator.

4. Transformation from polar coordinates space to conformal subdivision space $T_{\mathrm{P} d}:(\rho, \theta) \rightarrow \eta$ :

$$
\eta=\eta^{\mathrm{i}-\mathrm{S} d}+\left\{\begin{array}{c}
\rho \cos \theta \\
\rho \sin \theta
\end{array}\right\}
$$

where the Jacobian $J_{\mathrm{P} d}=\left|\partial\left(\eta_{1}, \eta_{2}\right) / \partial(\rho, \theta)\right|$ is:

$$
J_{\mathrm{P} d}=\rho
$$

As it is well-known, the resulting Jacobian is proportional to the radial coordinate $\rho$, and thus cancels out the weak singularity $1 / r$. The domain of polar coordinates is $(\rho, \theta) \in\left[0, \bar{\rho}^{\mathrm{S} d}\right] \times$ 


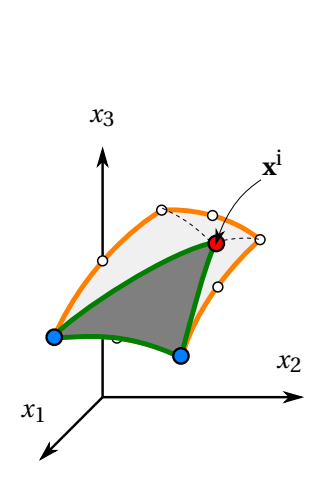

Element real space

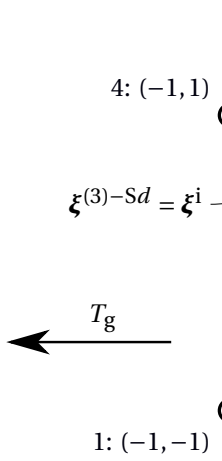

1: $(-1,-1)$

$\xi$

$\xi_{2}$

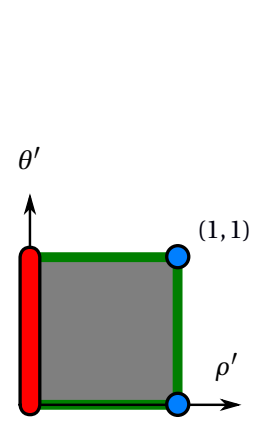

Polar coord. reference space

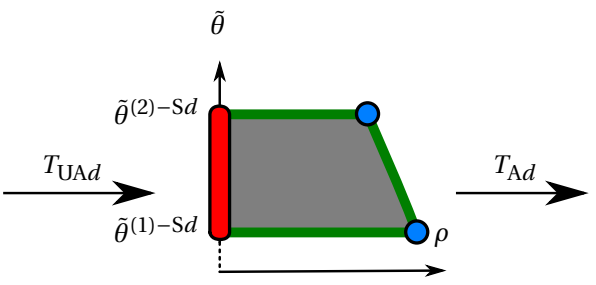

Altered polar coord. space
3: $(1,1)$

$\xi^{(2)-S d}$

$\xi_{1}$

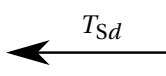

2: $(1,-1)$

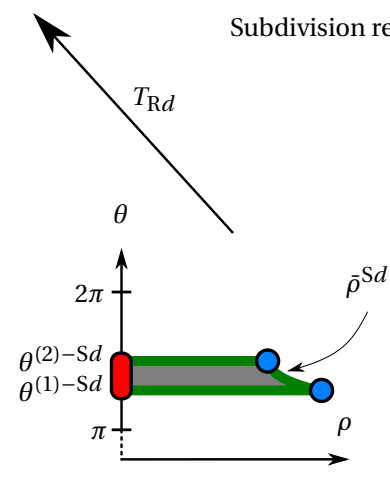

Polar coordinates space
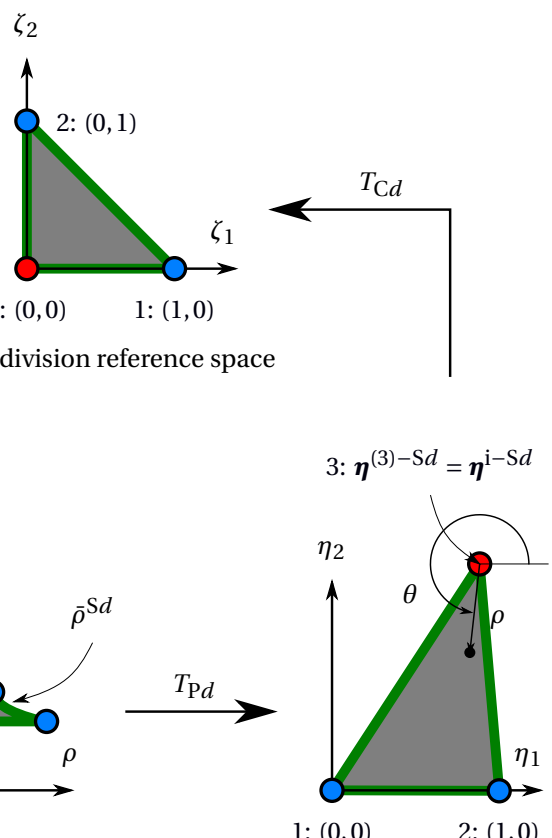

Figure 1: Proposed numerical scheme

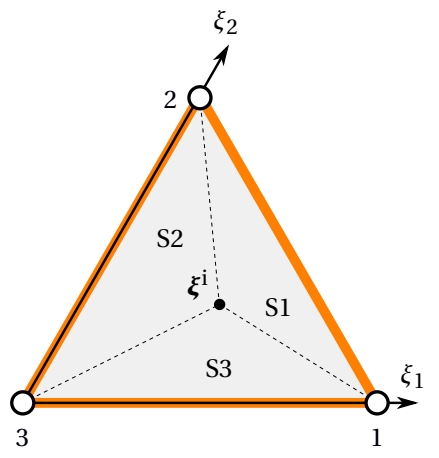

(a) Triangular elements

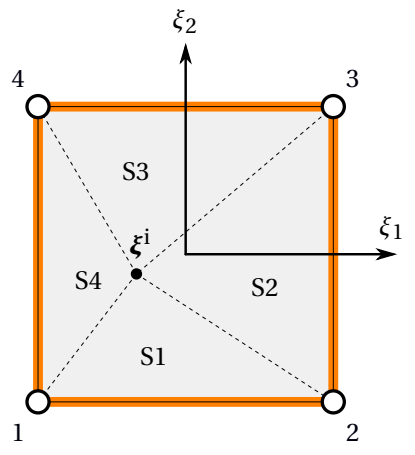

(b) Quadrilateral elements

Figure 2: Subdivision pattern

$\left[\theta^{(1)-\mathrm{S} d}, \theta^{(2)-\mathrm{S} d}\right]$, where:

$$
\begin{aligned}
& \bar{\rho}^{\mathrm{S} d}=-\frac{\eta_{2}^{\mathrm{i}-\mathrm{S} d}}{\sin \theta} \\
& \theta^{(1)-\mathrm{S} d}=\pi+\arccos \frac{\eta_{1}^{\mathrm{i}-\mathrm{S} d}}{\sqrt{\left(\eta_{1}^{\mathrm{i}-\mathrm{S} d}\right)^{2}+\left(\eta_{2}^{\mathrm{i}-\mathrm{S} d}\right)^{2}}} \\
& \theta^{(2)-\mathrm{S} d}=2 \pi-\arccos \frac{1-\eta_{1}^{\mathrm{i}-\mathrm{S} d}}{\sqrt{\left(1-\eta_{1}^{\mathrm{i}-\mathrm{S} d}\right)^{2}+\left(\eta_{2}^{\mathrm{i}-\mathrm{S} d}\right)^{2}}}
\end{aligned}
$$

It is also known that $\theta \in(\pi, 2 \pi), \sin \theta<0$ and $\eta_{2}^{\mathrm{i}-\mathrm{S} d}>0$ unless the subdivision degenerates, in which case the subdivision area is zero

and it does not contribute to the integral. At this point, it is possible to perform the classical Taylor expansion [8] of $r_{k}=x_{k}-x_{k}^{\mathrm{i}}$ at $\rho=0$ in terms of $\rho$ and $\theta$ :

$$
r_{k}=\rho\left(A_{k}+B_{k} \rho+\ldots\right)
$$

where $A_{k}=\left(\partial x_{k} / \partial \rho\right)^{\mathrm{i}}, B_{k}=\left(\partial^{2} x_{k} / \partial \rho^{2}\right)^{\mathrm{i}} / 2$, etc, and:

$$
\begin{aligned}
& A_{k}=\left(\frac{\partial x_{k}}{\partial \eta_{1}}\right)^{\mathrm{i}} \cos \theta+\left(\frac{\partial x_{k}}{\partial \eta_{2}}\right)^{\mathrm{i}} \sin \theta \\
& B_{k}=\frac{1}{2}\left(\frac{\partial^{2} x_{k}}{\partial \eta_{1}^{2}}\right)^{\mathrm{i}} \cos ^{2} \theta+\left(\frac{\partial^{2} x_{k}}{\partial \eta_{1} \partial \eta_{2}}\right)^{\mathrm{i}} \sin \theta \cos \theta \\
&+\frac{1}{2}\left(\frac{\partial^{2} x_{k}}{\partial \eta_{2}^{2}}\right)^{\mathrm{i}} \sin ^{2} \theta
\end{aligned}
$$

etc. Therefore, the distance expansion becomes:

$$
r=\sqrt{r_{k} r_{k}}=\rho \sqrt{A_{k} A_{k}+2 A_{k} B_{k} \rho+\ldots}
$$

where summation convention is implied. As a consequence, the distance has the following asymptotic behavior:

$$
\begin{aligned}
\lim _{\rho \rightarrow 0} r & =\rho \sqrt{A_{k} A_{k}} \\
& =\rho \sqrt{K_{11} \cos ^{2} \theta+K_{22} \sin ^{2} \theta+2 K_{12} \cos \theta \sin \theta}
\end{aligned}
$$

where constants $K_{i j}$ are obtained from:

$$
K_{i j}=\left(\frac{\partial x_{k}}{\partial \eta_{i}}\right)^{\mathrm{i}}\left(\frac{\partial x_{k}}{\partial \eta_{j}}\right)^{\mathrm{i}}=\left(\mathbf{T}_{i}^{\eta}\right)^{\mathrm{i}} \cdot\left(\mathbf{T}_{j}^{\eta}\right)^{\mathrm{i}}
$$




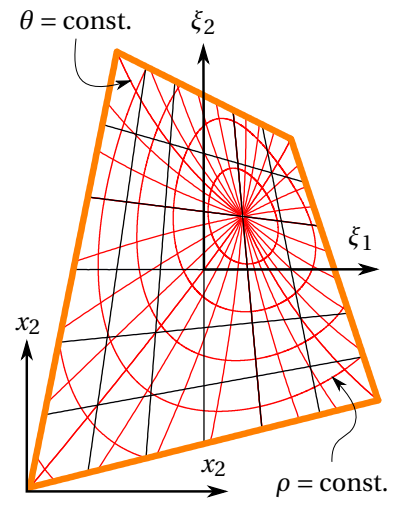

(a) Conventional polar transf.

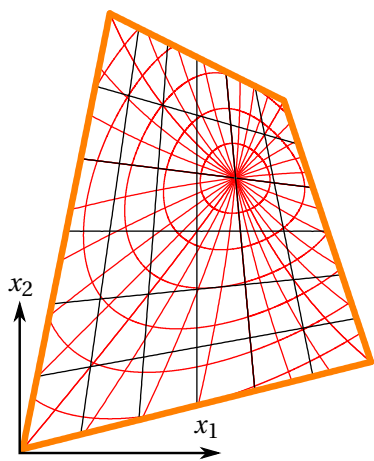

(b) Conformal polar transf.
Figure 3: Mapping of polar coordinates onto real space. Planar 4-node quadrilateral element with $\xi_{1}$ and $\xi_{2}$ isolines in black, and $\rho$ and $\theta$ isolines in red. Collocation point is located at $\xi^{i}=(1 / 3,1 / 3)$.

By squaring and reordering Eq. (18, the well-known oblique ellipse described by Guiggiani et al. [8] can be obtained. The canonical weak singularity $1 / r$ can be written as:

$$
\lim _{\rho \rightarrow 0} \frac{1}{r}=\frac{1}{\rho \sqrt{K_{11} \cos ^{2} \theta+K_{22} \sin ^{2} \theta+2 K_{12} \cos \theta \sin \theta}}
$$

which exhibits the previously mentioned secondary quasisingularities when $K_{11} \gg K_{22}$ or $K_{22} \gg K_{11}$ (high aspect ratios) or $\left|K_{12}\right| \gg 0$ (high skewness). However, since the conformality conditions from Eqs. (6) and (7) are imposed, it is straightforward to see respectively that $K_{11}=K_{22}$ and $K_{12}=0$. This means that the oblique ellipse described by Guiggiani et al. [8] is no longer an ellipse but a circle, which guarantees a shape independent cancellation of the weak singularity and the removal of secondary quasisingularities completely. Fig. 3 illustrates for a general planar 4node quadrilateral element how the limit expressed in Eq. 18 behaves for conventional (Fig. 3a) and conformal (Fig. 3p) polar transformations. In the latter case, lines of constant $\rho$ become circles as $\rho$ (and $r$ ) tends to zero, leading to a perfect mapping of polar coordinates onto the real space in the limit as $\rho \rightarrow 0$.

5. Transformation from altered polar coordinates to polar coordinates $T_{\mathrm{A} d}:(\rho, \tilde{\theta}) \rightarrow(\rho, \theta)$. It performs a non-linear transformation of the angular coordinate in order to treat the primary angular quasi-singularities produced at next and final transformation (transformation 6). The radial coordinate remains unaltered. At this point of the paper, it is considered as a generic transformation $\theta=\theta(\tilde{\theta})$ with Jacobian $J_{\mathrm{A} d}=\partial \theta / \partial \tilde{\theta}$, and domain $\tilde{\theta} \in\left[\tilde{\theta}^{(1)-S d}, \tilde{\theta}^{(2)-S d}\right]$. Previously proposed non-linear transformations like Khayat's transformation [13] or sigmoidal transformations [19, 15] can be used at this step (see A). In this paper, four robust and efficient novel transformations are proposed (see Section 3 .

6. Transformation from normalized polar coordinates to altered polar coordinates $T_{\mathrm{UAd}}:\left(\rho^{\prime}, \theta^{\prime}\right) \rightarrow(\rho, \tilde{\theta}):$

$$
\left\{\begin{array}{c}
\rho \\
\tilde{\theta}
\end{array}\right\}=\left\{\begin{array}{c}
0 \\
\tilde{\theta}^{(1)-\mathrm{S} d}
\end{array}\right\}+\left(\begin{array}{cc}
\bar{\rho}^{\mathrm{S} d} & 0 \\
0 & \Delta \tilde{\theta}^{\mathrm{S} d}
\end{array}\right)\left\{\begin{array}{c}
\rho^{\prime} \\
\theta^{\prime}
\end{array}\right\}
$$

where $\Delta \tilde{\theta}^{\mathrm{S} d}=\tilde{\theta}^{(2)-\mathrm{S} d}-\tilde{\theta}^{(1)-\mathrm{S} d}$, and the Jacobian of the transfor-

$$
\begin{aligned}
& \text { mation } J_{\mathrm{UA} d}=\left|\partial(\rho, \tilde{\theta}) / \partial\left(\rho^{\prime}, \theta^{\prime}\right)\right| \text { is: } \\
& J_{\mathrm{UA} d}=\bar{\rho}^{\mathrm{S} d} \Delta \tilde{\theta}^{\mathrm{S} d}=-\frac{\eta_{2}^{\mathrm{i}-\mathrm{S} d}}{\sin \theta} \Delta \tilde{\theta}^{\mathrm{S} d}
\end{aligned}
$$

The presence of $\sin \theta$ in the denominator produces the primary quasi-singularities, which are treated by the previous transformation 5. Eventually, a Gauss-Legendre product rule of $N_{\rho} \times N_{\theta}$ points is applied in this region $\left(\rho^{\prime}, \theta^{\prime}\right) \in[0,1] \times[0,1]$.

It is possible to condense transformations 2, 3 and 4 and use a straightforward transformation $T_{\mathrm{R} d}:(\rho, \theta) \rightarrow \xi$ :

$$
\xi=\xi^{\mathrm{i}}+\mathbf{S}^{\mathrm{S} d} \cdot \mathbf{C}^{\mathrm{S} d}\left\{\begin{array}{c}
\rho \cos \theta \\
\rho \sin \theta
\end{array}\right\}=\xi^{\mathrm{i}}+\mathbf{R}^{\mathrm{S} d}\left\{\begin{array}{c}
\rho \cos \theta \\
\rho \sin \theta
\end{array}\right\}
$$

with Jacobian proportional to the radial coordinate $\rho$ :

$J_{\mathrm{R} d}=\rho \frac{\left(\xi_{1}^{(1)-\mathrm{S} d}-\xi_{1}^{\mathrm{i}}\right)\left(\xi_{2}^{(2)-\mathrm{S} d}-\xi_{2}^{\mathrm{i}}\right)-\left(\xi_{1}^{(2)-\mathrm{S} d}-\xi_{1}^{\mathrm{i}}\right)\left(\xi_{2}^{(1)-\mathrm{S} d}-\xi_{2}^{\mathrm{i}}\right)}{\eta_{2}^{\mathrm{i}-\mathrm{S} d}}$

After applying all these transformations, the numerical integration of a weakly singular integrand $f \sim \mathscr{O}(1 / r)$ over a given element becomes:

$$
\begin{aligned}
I & =\int_{\Gamma} f \mathrm{~d} \Gamma=\sum_{d=1}^{N_{\mathrm{S}}} \int_{0}^{1} \int_{0}^{1} f\left(\rho^{\prime}, \theta^{\prime}\right) J_{\mathrm{g}}\left(\rho^{\prime}, \theta^{\prime}\right) J_{\mathrm{R} d} J_{\mathrm{A} d} J_{\mathrm{UA} d} \mathrm{~d} \rho^{\prime} \mathrm{d} \theta^{\prime} \\
& \approx \sum_{d=1}^{N_{\mathrm{S}}} \sum_{k_{\rho}=1}^{N_{\rho}} \sum_{k_{\theta}=1}^{N_{\theta}} f^{\left(k_{\rho}\right),\left(k_{\theta}\right)} J_{\mathrm{g}}^{\left(k_{\rho}\right),\left(k_{\theta}\right)} J_{\mathrm{R} d} J_{\mathrm{A} d} J_{\mathrm{UA} d} w^{\left(k_{\rho}\right)} w^{\left(k_{\theta}\right)}
\end{aligned}
$$

where $f^{\left(k_{\rho}\right),\left(k_{\theta}\right)}=f\left(\rho^{\prime\left(k_{\rho}\right)}, \theta^{\prime\left(k_{\theta}\right)}\right)$ and $J_{\mathrm{g}}^{\left(k_{\rho}\right),\left(k_{\theta}\right)}=J_{\mathrm{g}}\left(\rho^{\prime\left(k_{\rho}\right)},{\theta^{\prime}}^{\left(k_{\theta}\right)}\right)$. Coordinates and weights of radial and angular quadrature rules are respectively denoted as $\rho^{\prime\left(k_{\rho}\right)}, w^{\left(k_{\rho}\right)}$ and $\theta^{\prime\left(k_{\theta}\right)}, w^{\left(k_{\theta}\right)}$. The integration of any other singular integral with a higher singularity order can be performed via regularization before or after discretization, which turns the original singular integral into weakly singular surface integrals and quasi-singular line integrals. The most general method is perhaps the regularization after discretization, which was originally developed by Guiggiani et al [8, 9, 12,7]. A more elegant but more restricted method is the regularization before discretization, typically using simple solutions and Stokes' theorem, see e.g. [4, 6, 1].

\section{Non-linear transformations for pri- mary angular quasi-singularities}

As it was previously mentioned, primary angular quasi-singularities are those strong quasi-singularities emerging when the collocation point is near an element edge or vertex. This issue has been treated via $h$ (integration domain subdivision) and/or $p$ (adaptive quadrature rule) refinement [18], and non-linear transformations [13, 19, 15]. The $h$ - and $p$ refinement essentially treat the issue by increasing the number of integration points. The use of non-linear transformations is much more appropriate since a high reduction of the number of integration points may be achieved. However, their efficiency vary with the integrand since some excel with weakly singular kernels and others excel with hypersingular kernels (after regularization). In this section, four novel transformations are presented and analytically compared against others present in the literature, which have also been adapted to the present scheme (see A. Furthermore, more insight is given for explaining why there seems not to be a definitive non-linear transformation for all kinds all kernels.

In the proposed scheme, the primary quasi-singularities appear in the Jacobian $J_{\mathrm{UA} d}$, see Eq. 22. In particular, they are produced by the 


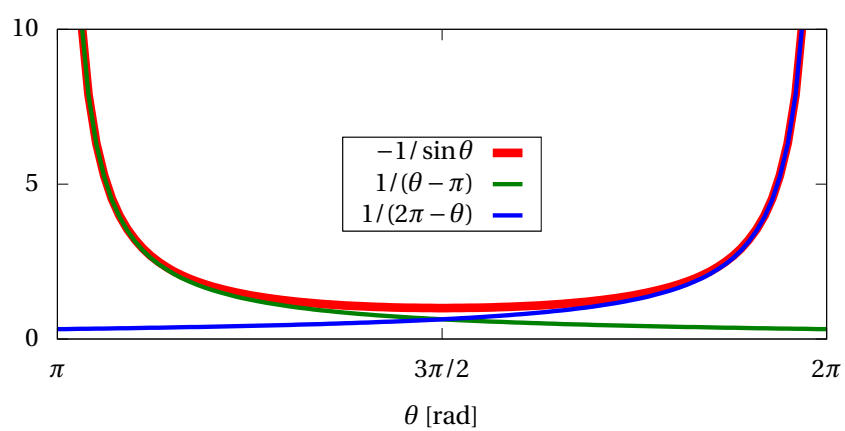

Figure 4: Primary angular quasi-singularities

factor $-1 / \sin \theta$, which, taking into account that $\theta \in(\pi, 2 \pi)$, contain strong quasi-singularities that are located $\theta=\pi$ and $\theta=2 \pi$, see Fig. 4

These singularities are fixed in the $\theta$ space, and thus they becomes more relevant as the integration limits $\theta^{(1)-\mathrm{S} d} \rightarrow \pi^{+}$and/or $\theta^{(2)-\mathrm{S} d} \rightarrow$ $(2 \pi)^{-}$, i.e. as the location of the collocation point approach a vertex (only one quasi-singularity emerges) or edge (quasi-singularities at both ends emerge). Fig. 5 shows how the location of the singularity in the conformal subdivision space $\left(\eta^{\mathrm{i}-\mathrm{S} d}\right)$ influences the relevance of primary quasi-singularities.

There exists a transformation that exactly cancels out these quasisingularities, and it appeared in Khayat et al. [13], although it is not clear if they were aware of this fact. Khayat's transformation is here denoted as the arctan exp transformation, and it has been adapted to the present scheme, see A It also has the remarkable property of transforming shape functions into combinations of hyperbolic functions, which leads to integrands of more simple analytical form than other transformations. This transformation is optimal in the sense of removing these quasi-singularities, and it is a priori the ultimate choice for this purpose.

Rong et al. [19] proposed the use of a particular family of sigmoidal functions due to Johnston [11]. They are $[0,1]$ onto $[0,1]$ transformations which concentrate integration points at $\theta=\pi$ and $\theta=2 \pi$. Sigmoidal transformations of order 2 (denoted as sig2 here) and 3 (denoted as sig3 here) have been adapted to the present scheme, see A As Rong et al. [19] and $L v$ et al. [15] shown, they work very well in practice, being sig3 the best for weakly singular kernels and sig 2 the best for hypersingular kernels. Rong et al. [19] also reported that their experience showed that Khayat's transformation performs worse than their proposed sigmoidal transformations, especially for hypersingular kernels. Nonetheless, results supporting and discussing this at first surprising behavior were not presented. Our results (Section 4) confirm Rong et al. [19] findings. Interestingly, in the context of BEM quasi-singular integrals, Ma and Kamiya [16] also found that the exact distance transformation for hypersingular kernels underperforms the use of other weaker distance transformation applied to hypersingular kernels.

The main difference between weakly singular, strongly singular and hypersingular kernels (after regularization) is their degree of complexity. If these kernels are expressed in terms of Guiggiani's polar expansions, e.g. through Eq. (14) and so forth, it is clear that kernels become increasingly more intricate rational functions with higher powers of trigonometric functions of $\theta$ as the main ingredient. Therefore, when the whole integrand in terms of quadrature coordinates $\rho^{\prime}$ and $\theta^{\prime}$ is considered, it becomes increasingly more important how smoothly the non-linear transformation transforms trigonometric functions and kernels than how it is able to cancel the mentioned angular quasi-singularities.

Figs. 6 and 7 show how each of the previous transformations works for two critical locations of the collocation point $\eta^{\mathrm{i}}=(0.50,0.01)$ and $\eta^{\mathrm{i}}=(0.50,0.00001)$. For now, lets consider only linear, arctan exp, sig2 and sig 3 transformations, and ignore the rest. The first column shows the angular coordinate transformation $\theta\left(\theta^{\prime}\right)$, the second column shows the product of the quasi-singular term $-1 / \sin \theta$ and the Jacobian of the
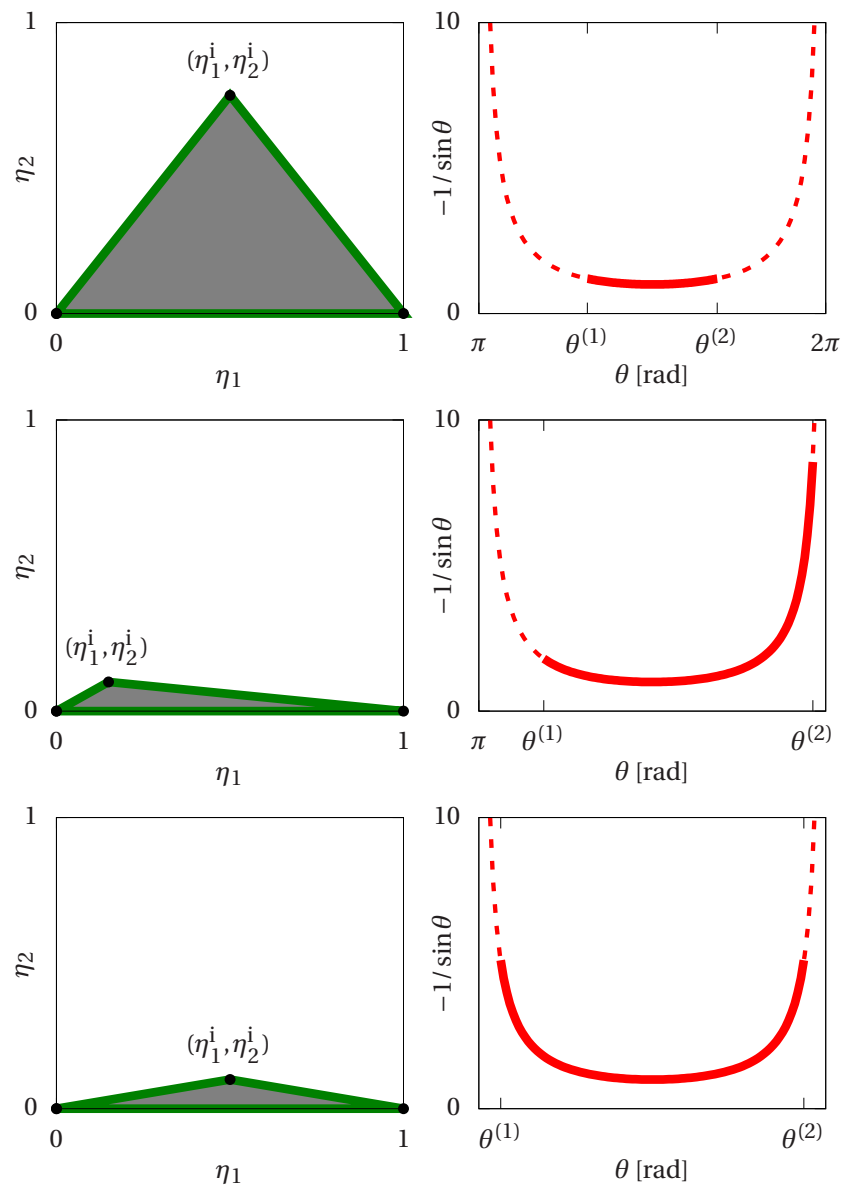

Figure 5: Influence of $\eta^{\mathrm{i}-\mathrm{S} d}$ on the relevance of primary quasisingularities. Top: non-relevant primary quasi-singularities $\left(\eta^{\mathrm{i}}=(0.50,0.75)\right)$. Center: one-end primary quasi-singularity $\left(\eta^{\mathrm{i}}=(0.15,0.10)\right)$. Bottom: two-end primary quasi-singularities $\left(\eta^{\mathrm{i}}=(0.50,0.10)\right)$.

transformation $\left(J_{\mathrm{A}}\right)$ normalized by its value at $\theta^{\prime}=0.5$, and the third and fourth columns how $\sin \theta$ and $\cos \theta$ are transformed. It becomes very clear that the exact cancellation of primary quasi-singularities via the arctan exp transformation implies a drastic distortion of trigonometric functions. It turns the sine function into a peak-like function, and the cosine function into a step-like function. Sigmoidal transformations weaken the quasi-singularities without completely removing them. In fact, by developing these transformations (see A, it is possible to show that in terms of the altered angular coordinate $\theta$ :

$$
\lim _{\tilde{\theta} \rightarrow \mp 1} \frac{-1}{\sin \theta} J_{\mathrm{A}}=\frac{m}{1 \pm \tilde{\theta}}
$$

where $m=2,3$ is the transformation order. This demonstrates that the strong quasi-singularities remain there, although they have been moved away. Despite not canceling the quasi-singularities, the resulting trigonometric functions are smoother than those resulting from the arctan exp transformation. Also, it is observed that sig2 transformation is smoother than sig3, which justifies why it works better with hypersingular kernels where trigonometric functions become more relevant. These results show the need of finding more transformations with different tradeoffs between both factors, but in any case completely free from quasisingularities.

With this idea in mind, an exhaustive search of alternative sigmoid functions whose Jacobians smoothen quasi-singularities to a different extent has been performed. From this search, several novel transfor- 

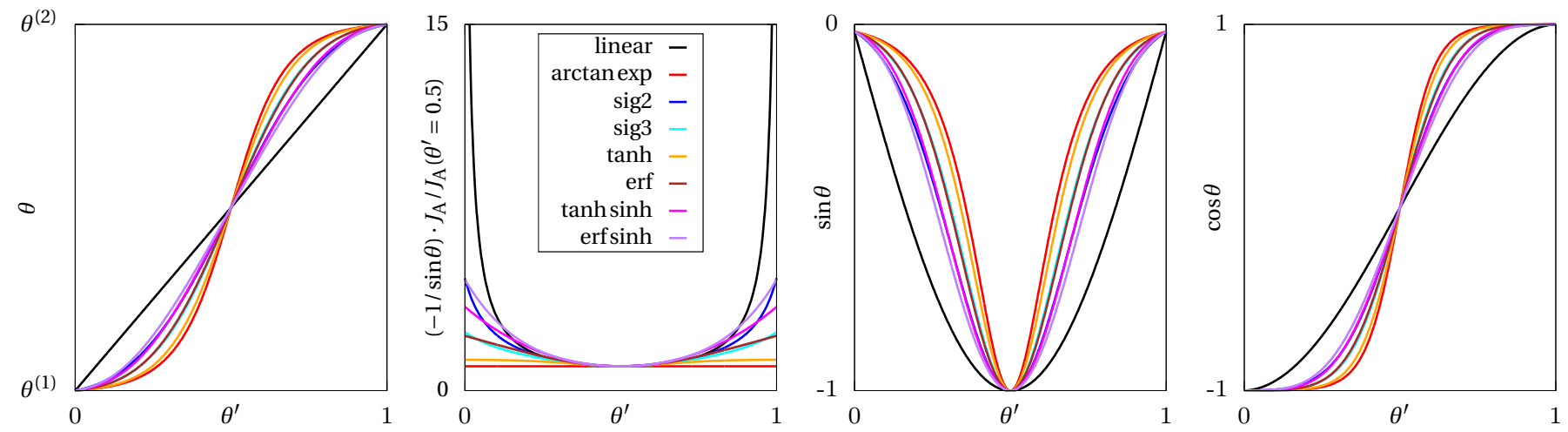

Figure 6: Effects of non-linear transformations on the product of Jacobians $J_{\mathrm{A}} J_{\mathrm{UA}}$ and trigonometric functions $\left(\eta^{\mathrm{i}}=(0.50,0.01)\right)$
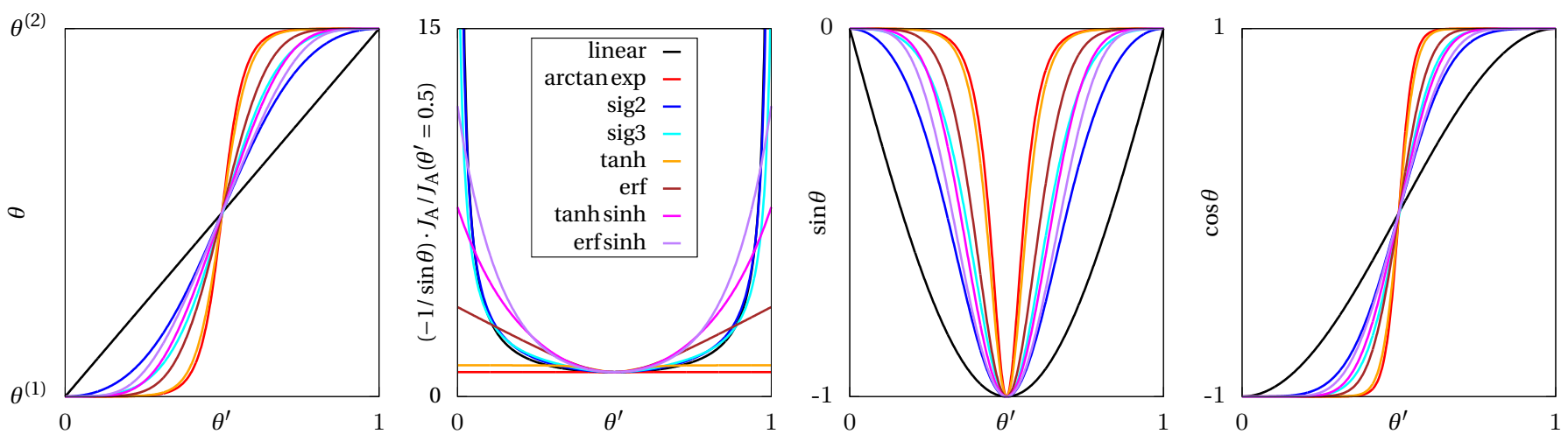

Figure 7: Effects of non-linear transformations on the product of Jacobians and trigonometric functions $\left(\eta^{\mathrm{i}}=(0.50,0.00001)\right)$

mations able to consistently achieve better performance than sigmoidal functions have been found.

\section{1 tanh transformation}

It is considered a Jacobian of the form:

$$
J_{\mathrm{A}}=(\theta-\pi)(2 \pi-\theta)
$$

which contain the two zeros that cancel out the strong singularities of the quasi-singular term $-1 / \sin \theta$. The transformation which produces such a Jacobian is a hyperbolic tangent transformation (tanh transformation):

$$
\theta=\frac{3 \pi}{2}+\frac{\pi}{2} \tanh \left(\frac{\pi}{2} \tilde{\theta}\right)
$$

where $\lim _{\tilde{\theta} \rightarrow \pm \infty} \theta(\tilde{\theta})=3 \pi / 2 \pm \pi / 2$. In terms of the altered angular coordinate $\tilde{\theta}$, the product of the quasi-singular term and the Jacobian $J_{\mathrm{A}}$ can be written as:

$$
\frac{-1}{\sin \theta} J_{\mathrm{A}}=\frac{-1}{\sin (3 \pi / 2+\pi / 2 \cdot \tanh (\pi / 2 \cdot \tilde{\theta}))} \frac{\pi^{2}}{4 \cosh (\pi / 2 \cdot \tilde{\theta})^{2}}
$$

whose limit when $\tilde{\theta} \rightarrow \pm \infty$ is:

$$
\lim _{\tilde{\theta} \rightarrow \pm \infty} \frac{-1}{\sin \theta} J_{\mathrm{A}}=\pi
$$

showing a full removal of the singularities in the altered angular coordinate space. This asymptotic behavior is shown in Fig. 8 Since the hyperbolic tangent function is a rescaled version of the well-known logistic function $(1+\exp (-x))^{-1}$, the transformation can also be written in terms of this.

\section{2 erf transformation}

It is considered a transformation based on another well-known sigmoid function, the error function, which leads to the following error function transformation (erf transformation):

$$
\theta=\frac{3 \pi}{2}+\frac{\pi}{2} \operatorname{erf} \tilde{\theta}
$$

where $\lim _{\tilde{\theta} \rightarrow \pm \infty} \theta(\tilde{\theta})=3 \pi / 2 \pm \pi / 2$. This transformation asymptotically removes the singularities at $\theta=\pi$ and $\theta=2 \pi$. This is demonstrated by expanding $(-1 / \sin \theta) \cdot J_{\mathrm{A}}$ in the limit $\tilde{\theta} \rightarrow \pm \infty$ :

$$
\begin{aligned}
\frac{-1}{\sin \theta} J_{\mathrm{A}} & =-\frac{\sqrt{\pi} e^{-\tilde{\theta}^{2}}}{\sin (3 \pi / 2+\pi / 2 \cdot \operatorname{erf} \tilde{\theta})} \\
\lim _{\tilde{\theta} \rightarrow \pm \infty} \frac{-1}{\sin \theta} J_{\mathrm{A}} & = \pm 2 \tilde{\theta}
\end{aligned}
$$

which are plotted in Fig. 8

\section{3 tanh sinh transformation}

By inserting the sinh function inside the tanh transformation, the following transformation is achieved (tanh sinh transformation):

$$
\theta=\frac{3 \pi}{2}+\frac{\pi}{2} \tanh (\sinh \tilde{\theta})
$$

where $\lim _{\tilde{\theta} \rightarrow \pm \infty} \theta(\tilde{\theta})=3 \pi / 2 \pm \pi / 2$. This type of transformation is also called a Double-Exponential (DE) transformation [17], and it has a long history for evaluating integrals using the trapezoidal formula in a very efficient manner and with arbitrary precision. When it is applied to the 

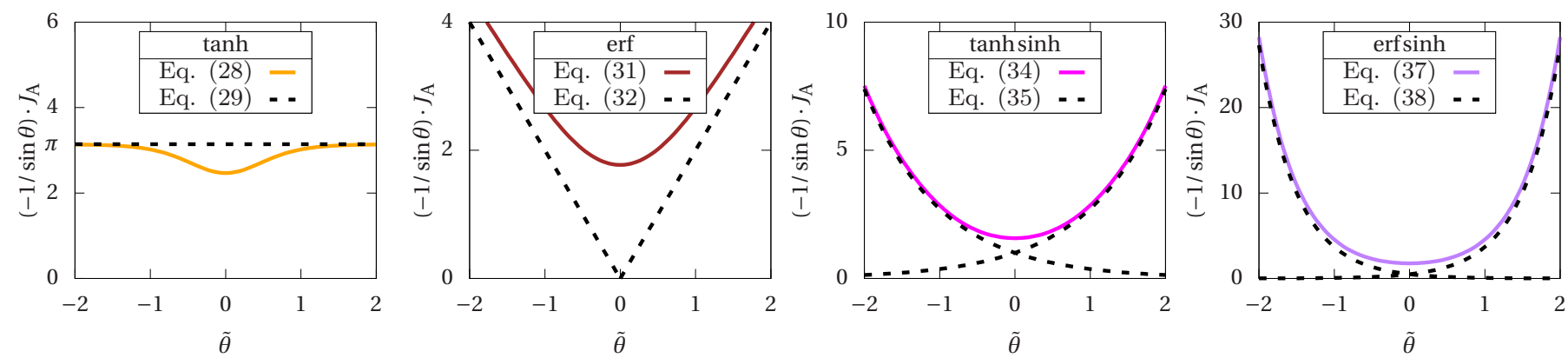

Figure 8: Asymptotic behavior of tanh, erf, tanh sinh and erf $\sinh$ transformations in $(-1 / \sin \theta) \cdot J_{\mathrm{A}}$.

present problem, it is found that singularities are removed with the following asymptotics:

$$
\begin{aligned}
\frac{-1}{\sin \theta} J_{\mathrm{A}} & =\frac{\pi}{2} \frac{\cosh \tilde{\theta}}{[\cosh (\sinh \tilde{\theta})]^{2} \cos \left(\frac{\pi}{2} \tanh (\sinh \tilde{\theta})\right)} \\
\lim _{\tilde{\theta} \rightarrow \pm \infty} \frac{-1}{\sin \theta} J_{\mathrm{A}} & =e^{ \pm \tilde{\theta}}
\end{aligned}
$$

where the exponential asymptotic behavior is not problematic since for double precision calculations $\theta(\tilde{\theta})-\pi<10^{-15}$ and $2 \pi-\theta(\tilde{\theta})<$ $10^{-15}$ are obtained respectively at $\tilde{\theta} \approx-3.5$ and $\tilde{\theta} \approx 3.5$, and thus $(-1 / \sin \theta) \cdot J_{\mathrm{A}}$ is kept in a well-behaved domain. Fig. 8 illustrates Eqs. (34) and 35 , where it can be observed that the resulting $(-1 / \sin \theta) \cdot J_{\mathrm{A}}$ is completely free from end-point singularities.

\section{4 erf sinh transformation}

By inserting the sinh function inside the erf transformation, the following transformation is proposed (erf sinh transformation):

$$
\theta=\frac{3 \pi}{2}+\frac{\pi}{2} \operatorname{erf}(\sinh \tilde{\theta})
$$

where $\lim _{\tilde{\theta} \rightarrow \pm \infty} \theta(\tilde{\theta})=3 \pi / 2 \pm \pi / 2$. This transformation also removes the singularities and it has the following asymptotic behavior:

$$
\begin{aligned}
\frac{-1}{\sin \theta} J_{\mathrm{A}} & =-\frac{\sqrt{\pi} e^{-(\sinh \tilde{\theta})^{2}} \cosh \tilde{\theta}}{\sin (3 \pi / 2+\pi / 2 \cdot \operatorname{erf}(\sinh \tilde{\theta}))} \\
\lim _{\tilde{\theta} \rightarrow \pm \infty} \frac{-1}{\sin \theta} J_{\mathrm{A}} & =\frac{1}{2} e^{ \pm 2 \tilde{\theta}}
\end{aligned}
$$

which is illustrated in Fig. 8 As in the previous case, the exponential asymptotic behavior is not problematic since for double precision calculations $\theta(\tilde{\theta})-\pi<10^{-15}$ and $2 \pi-\theta(\tilde{\theta})<10^{-15}$ are obtained respectively at $\tilde{\theta} \approx-2.4$ and $\tilde{\theta} \approx 2.4$, and thus $(-1 / \sin \theta) \cdot J_{\mathrm{A}}$ is wellbehaved.

The five transformations arctanexp, tanh, erf, tanh sinh and erf sinh differ in how strictly they smooth out the problematic $-1 / \sin \theta$ term. All these transformations remove the quasi-singularities. In particular, arctan exp transformation produces an exact cancellation, tanh transformation produces an asymptotically exact cancellation (horizontal asymptote), erf transformation leaves a linear term (oblique asymptote), and tanh sinh and erf sinh transformations leave an exponential term (exponential asymptotics). In this sense, they increasingly relax how good is the cancellation of this problematic term. Now, if Figs. 6 and 7 are observed again, it can be seen than the effects of these new transformations are somehow in between the arctan exp and sig 2 transformations.

The most remarkable difference of these new transformations is that they remove the quasi-singularities (like the arctanexp transformation) while at the same time produce transformed trigonometric functions smoother than the arctan exp transformation (like sigmoidal functions).

\section{Results and discussion}

In this section, the numerical integration scheme proposed in Section 2 and the non-linear transformations presented in Section 3 are used to show the robustness and efficiency of the proposed methodology.

\subsection{Triangular elements}

In this section, the proposed algorithm is used to solve the example proposed by Rong et al. [19] for triangular elements. Their results are obtained by using sigmoidal transformations, while in the present work also the four transformations proposed in Section 3 are used.

The original example studies the influence of the collocation point location, element aspect ratio and type of kernel (weakly singular and hypersingular) for a quadratic triangular element extracted from a cylindrical surface. Four collocation points are considered: $a) \xi^{\mathrm{i}}=(0.3,0.3)$ (approximately in the center), $b$ ) $\xi^{\mathrm{i}}=(0.1,0.8)$ (near a vertex), $\left.c\right) \xi^{\mathrm{i}}=$ $(0.45,0.45)$ (near an edge) and $d$ ) $\xi^{\mathrm{i}}=(0.64,0.31)$ (very close to an edge). The element aspect ratio is controlled via the length $s$, which for $s=0.5$ leads to a triangle with an aspect ratio near 1 and for $s=10$ leads to an aspect ratio approximately 10. Fig. 9 shows the resulting parametrized triangular element. Four integrals are being considered in the present paper. The weakly singular integrals $I_{\mathrm{H}}$ and $I_{\mathrm{G}}$ are taken from the Singular Boundary Integral Equation for scalar wave propagation:

$$
\begin{aligned}
& I_{\mathrm{H}}=\int_{\Gamma} \frac{-e^{-i k r}}{4 \pi}\left(\frac{1}{r^{2}}+\frac{i k}{r}\right) \frac{\partial r}{\partial n} \phi \mathrm{d} \Gamma \\
& I_{\mathrm{G}}=\int_{\Gamma} \frac{e^{-i k r}}{4 \pi} \frac{1}{r} \phi \mathrm{d} \Gamma
\end{aligned}
$$

where $i$ is the imaginary unit, $k$ is the wavenumber, and $\phi$ is a shape function (in this example is $\phi=\xi_{2}^{2}$ ). The hypersingular integral $I_{\mathrm{M}}$ and weakly singular integral $I_{\mathrm{L}}$ are taken from the Hypersingular Boundary Integral Equation for scalar wave propagation:

$$
\begin{aligned}
& I_{\mathrm{M}}=\int_{\Gamma} \frac{e^{-i k r}}{4 \pi}\left[\left(\frac{3}{r^{3}}+\frac{3 i k}{r^{2}}+\frac{(i k)^{2}}{r}\right) \frac{\partial r}{\partial n} \frac{\partial r}{\partial n^{\mathrm{i}}}\right.\left.+\left(\frac{1}{r^{3}}+\frac{i k}{r^{2}}\right)\left(\mathbf{n} \cdot \mathbf{n}^{\mathrm{i}}\right)\right] \phi \mathrm{d} \Gamma \\
& I_{\mathrm{L}}=\int_{\Gamma} \frac{-e^{-i k r}}{4 \pi}\left(\frac{1}{r^{2}}+\frac{i k}{r}\right) \frac{\partial r}{\partial n^{\mathrm{i}}} \phi \mathrm{d} \Gamma
\end{aligned}
$$

where $\mathbf{n}$ is the unit normal at the observation point and $\mathbf{n}^{\mathrm{i}}$ is the unit normal at the collocation point. The hypersingular integral $I_{M}$ is regularized via Guiggiani's method [9].

Figs. 10 and 11 show the convergence of the proposed numerical integration scheme when evaluating $I_{\mathrm{G}}$ and $I_{\mathrm{M}}$ for $s=0.5$, collocation 


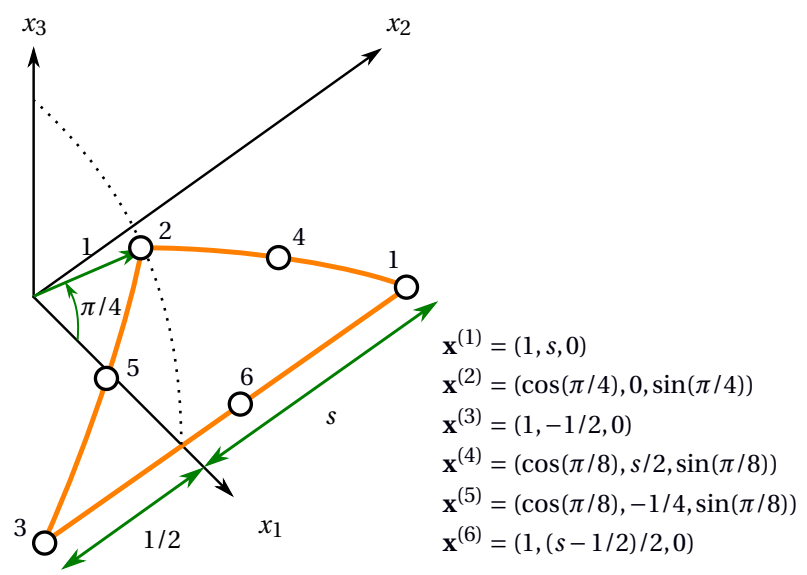

Figure 9: Parametrized triangular element extracted from cylindrical surface

points $a$ to $d$ and wavenumber $k=2$. Ordinates represent the relative error $\varepsilon=\left|I^{\text {num }}-I^{\mathrm{ref}}\right| /\left|I^{\mathrm{ref}}\right|$ in a logarithmic scale, where $I^{\text {num }}$ is evaluated using $N_{\theta}$ integration points in the angular coordinate and $N_{\rho}=6$ integration points in the radial coordinate, and $I^{\text {ref }}$ is evaluated using $N_{\theta}=N_{\rho}=32$ with tanh sinh transformation. For each case, the obtained convergence curves for linear transformation (no treatment for the primary quasi-singularities), and non-linear transformations arctan exp, sig2, sig3, tanh, erf, tanh sinh and erf sinh are shown using solid lines. Results obtained by Rong et al. [19] are indicated by markers and they show good agreement with the results presented in this paper. In all cases, the conformality conditions at the collocation point are enforced, so that secondary quasi-singularities are completely removed.

Fig. 10 shows the convergence results for the integral $I_{\mathrm{G}}$. It can be seen that all non-linear transformations drastically reduce the required $N_{\theta}$ for a given relative error, especially for collocation points near the element boundary (collocation points $b, c$ and $d$ ). However, it is shown that arctan exp and tanh transformations excels in this regard, although tanh one is somewhat better. It is also observed that sig 3 and erf transformations have virtually the same convergence rates for all collocation points, while erf sinh and sig2 transformations are clearly the worse. As expected, it can be seen that differences among non-linear transformations increases as the collocation point is closer to the element boundary.

Fig. 11] shows the convergence results for the integral $I_{\mathrm{M}}$. It is observed that all non-linear transformations significantly reduces the required $N_{\theta}$ for a given relative error. However, this reduction is smaller than for the integral $I_{\mathrm{G}}$. This result was explained in Section 3 where it was argued that the integration of hypersingular kernels is more sensitive to how trigonometric functions are transformed than in the cases of weakly singular and strongly singular kernels. In this sense, the optimal transformation in removing the primary quasi-singularities, i.e. the arctan exp transformation, is also the transformation that lose more performance, and is not much better than the linear transformation. This result is in agreement with what Rong et al. [19] reported. The tanh transformation also goes from being the best for $I_{\mathrm{G}}$ to be the second worse for $I_{\mathrm{M}}$. Unlike for $I_{\mathrm{G}}$, sig2 transformation performs somewhat better than sig 3 transformation for $I_{\mathrm{M}}$. Finally, it is observed that the efficacy of erf transformation is consistently similar to that of the sig2 transformation. tanh sinh transformation achieves the best convergence, while erf sinh becomes the second best.

In order to study the sensitivity to the element aspect ratio, Table 1 shows the required number of integration points in the angular direction $N_{\theta}$ for different values of $s$ (aspect ratio of the element) in order to achieve a relative error $\varepsilon=10^{-8}$. The collocation point $a$ is used, and the considered wavenumber is $k=0$. The column on the right indicates the

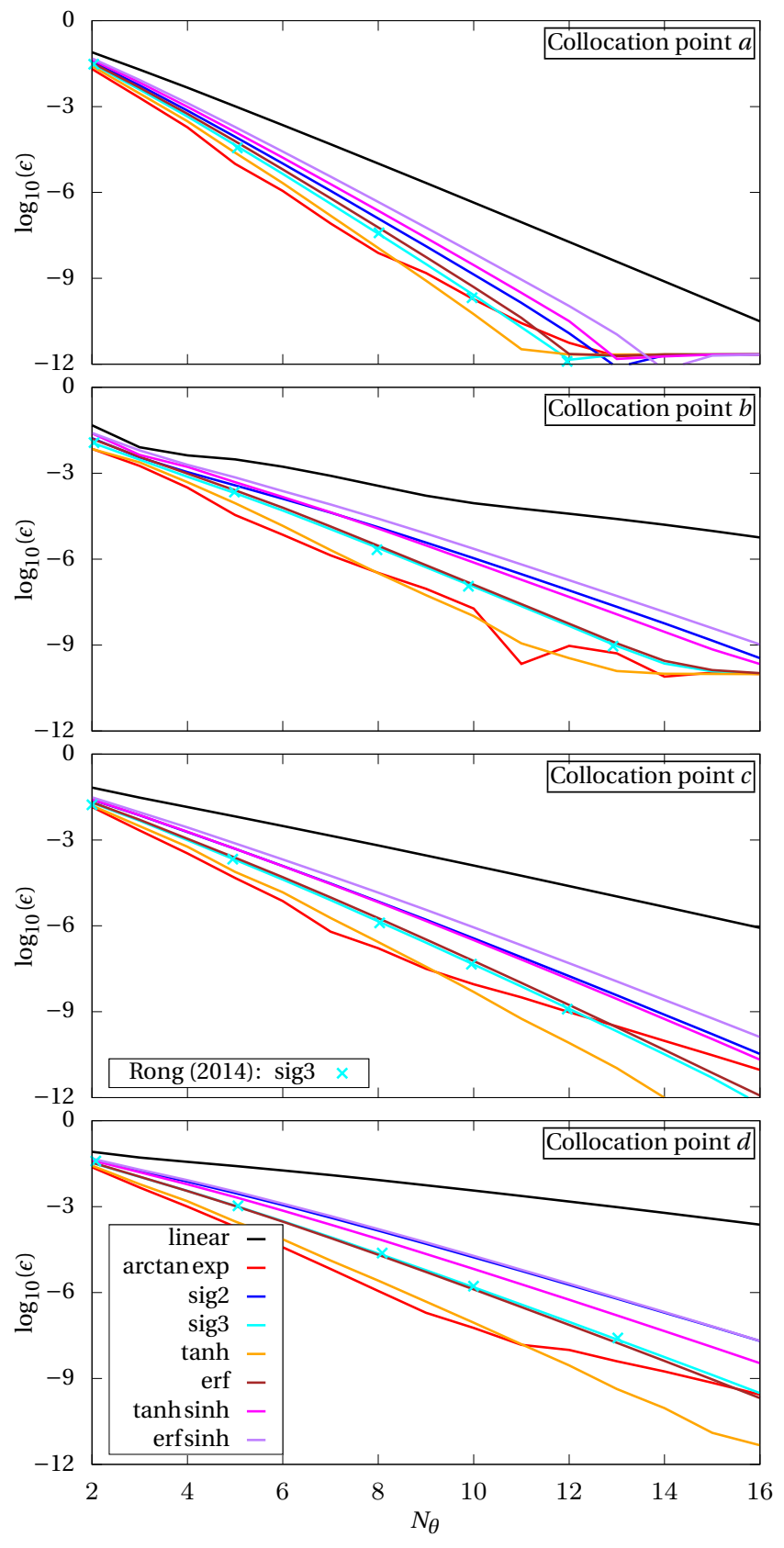

Figure 10: Convergence comparison for $I_{\mathrm{G}}(s=0.5$, collocation points $a, b, c, d$ and wavenumber $k=2$ ) 

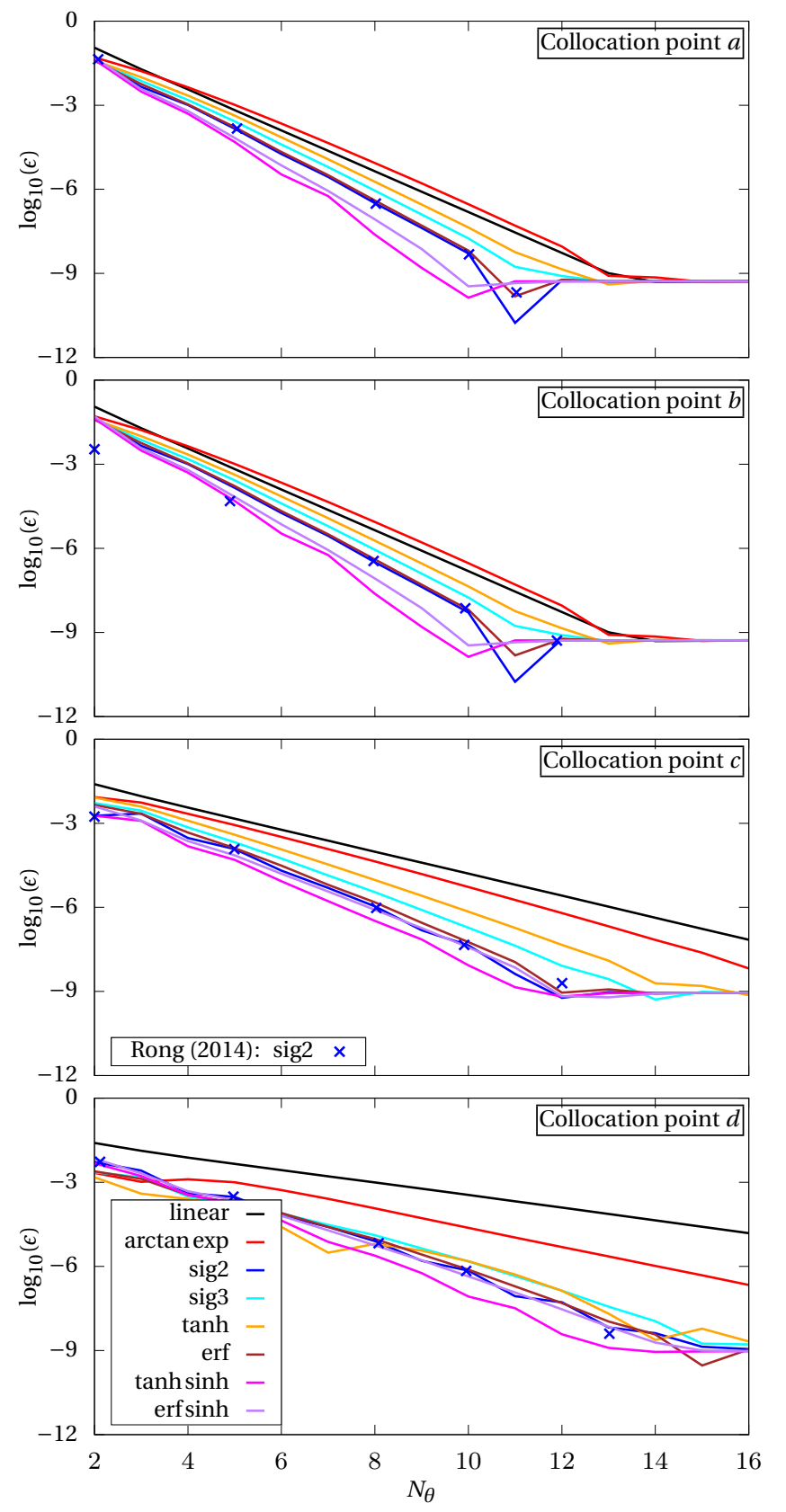

Figure 11: Convergence comparison for $I_{\mathrm{M}}(s=0.5$, collocation points $a, b, c, d$ and wavenumber $k=2$ )

\begin{tabular}{|c|c|c|c|c|c|c|c|}
\hline & $N_{\theta}$ & $s=0.5$ & $s=1.5$ & $s=2$ & $s=4$ & $s=10$ & Avg. \\
\hline \multirow{7}{*}{$I_{\mathrm{G}}$} & sig2 & 9 & 10 & 11 & 14 & 18 & 12.4 \\
\hline & $\operatorname{sig} 3$ & 8 & 9 & 9 & 11 & 14 & 10.2 \\
\hline & $\arctan \exp$ & 8 & 9 & 11 & 14 & 16 & 11.6 \\
\hline & $\tanh$ & 7 & 8 & 8 & 11 & 14 & 9.6 \\
\hline & erf & 8 & 9 & 10 & 11 & 13 & 10.2 \\
\hline & $\tanh \sinh$ & 9 & 10 & 11 & 13 & 15 & 11.6 \\
\hline & erf sinh & 9 & 11 & 12 & 14 & 17 & 12.6 \\
\hline \multirow{7}{*}{$I_{\mathrm{H}}$} & sig2 & 8 & 9 & 9 & 12 & 15 & 10.6 \\
\hline & sig3 & 9 & 10 & 11 & 13 & 18 & 12.2 \\
\hline & $\arctan \exp$ & 10 & 14 & 16 & 18 & 25 & 16.6 \\
\hline & $\tanh$ & 9 & 11 & 12 & 15 & 19 & 13.2 \\
\hline & erf & 8 & 10 & 10 & 13 & 16 & 11.4 \\
\hline & $\tanh \sinh$ & 8 & 9 & 9 & 11 & 12 & 9.8 \\
\hline & erf sinh & 8 & 9 & 10 & 12 & 13 & 10.4 \\
\hline \multirow{7}{*}{$I_{\mathrm{L}}$} & sig2 & 9 & 9 & 10 & 12 & 15 & 11.0 \\
\hline & sig3 & 9 & 10 & 11 & 13 & 18 & 12.2 \\
\hline & $\arctan \exp$ & 10 & 14 & 15 & 18 & 25 & 16.4 \\
\hline & $\tanh$ & 9 & 11 & 11 & 15 & 19 & 13.0 \\
\hline & erf & 8 & 10 & 10 & 13 & 16 & 11.4 \\
\hline & $\tanh \sinh$ & 9 & 9 & 10 & 11 & 13 & 10.4 \\
\hline & erf sinh & 9 & 10 & 11 & 12 & 14 & 11.2 \\
\hline \multirow{7}{*}{$I_{\mathrm{M}}$} & sig2 & 11 & 12 & 12 & 13 & 17 & 13.0 \\
\hline & sig3 & 11 & 13 & 14 & 16 & 20 & 14.8 \\
\hline & $\arctan \exp$ & 13 & 17 & 19 & 21 & 29 & 19.8 \\
\hline & $\tanh$ & 12 & 14 & 16 & 19 & 22 & 16.6 \\
\hline & erf & 11 & 11 & 12 & 14 & 18 & 13.2 \\
\hline & $\tanh \sinh$ & 9 & 9 & 10 & 11 & 14 & 10.6 \\
\hline & erf sinh & 8 & 8 & 10 & 11 & 13 & 10.0 \\
\hline
\end{tabular}

Table 1: Required $N_{\theta}$ to make relative error below $10^{-8}$ (collocation point $a$ and wavenumber $k=0$ )

average $N_{\theta}$ from the previous columns, and the overall best non-linear transformation is in bold. It is observed that the previously described performance rank order of non-linear transformations is more or less the same. The tanh transformation is the best for $I_{\mathrm{G}}$, tanh sinh transformation is the best for $I_{\mathrm{H}}$ and $I_{\mathrm{L}}$, and the erf sinh transformation is now the best for $I_{\mathrm{M}}$. For $I_{\mathrm{G}}$ it is obtained that transformations smoother than tanh increasingly requires more $N_{\theta}$. On the other hand, for $I_{M}$ it is seen that transformations smoother than tanh increasingly requires less $N_{\theta}$. For $I_{\mathrm{H}}$ and $I_{\mathrm{L}}$ the optimal transformation is tanh sinh, and $N_{\theta}$ increases for smoother and less smoother transformations.

\subsection{Quadrilateral elements}

In this section, the proposed algorithm is used to solve the example proposed by Lv et al. [15] for quadrilateral elements. Their results are obtained by using sigmoidal transformations, while in this work also the four transformations proposed in Section 3 are considered. Integrals $I_{\mathrm{G}}, I_{\mathrm{H}}, I_{\mathrm{L}}$ and $I_{\mathrm{M}}$ from Section 4.1 are again considered, but it is assumed that $k=0$ and $\phi=1$ in all cases. These integrals are evaluated over a highly distorted eight-node quadrilateral with an aspect ratio of approximately 4 (more details can be found in [15]).

Fig. 12 shows the relative error when evaluating $I_{\mathrm{G}}, I_{\mathrm{H}}, I_{\mathrm{L}}$ and $I_{\mathrm{M}}$ for collocation points located along a line from $\xi^{\mathrm{i}}=(0,0)$ (element center) to $\xi^{\mathrm{i}}=(1,1)$ (element vertex) with $\xi_{1}^{\mathrm{i}}=\xi_{2}^{\mathrm{i}}=\xi^{\mathrm{i}}$. The number of integration points for each triangular subdivision is fixed to $N_{\theta}=N_{\rho}=10$. It is observed that there is a strong dependency of the relative error on the location of the collocation point. Peaks and notches of relative error occur at certain locations which may vary with the non-linear transformation used. Integral $I_{\mathrm{G}}$ was also solved by Lv et al. [15] using sigmoidal transformations, and it can be seen that there is a close agreement between both results. As in the previous case, it is observed that tanh transformation is generally better than the exact arctan exp transformation, which is the worst in all cases except for $I_{\mathrm{G}}$. However, it is not seen that the tanh transformation excels for the weakly singular kernel $\left(I_{\mathrm{G}}\right)$, perhaps due to the absence of the shape function in the integrand. The theoretical 

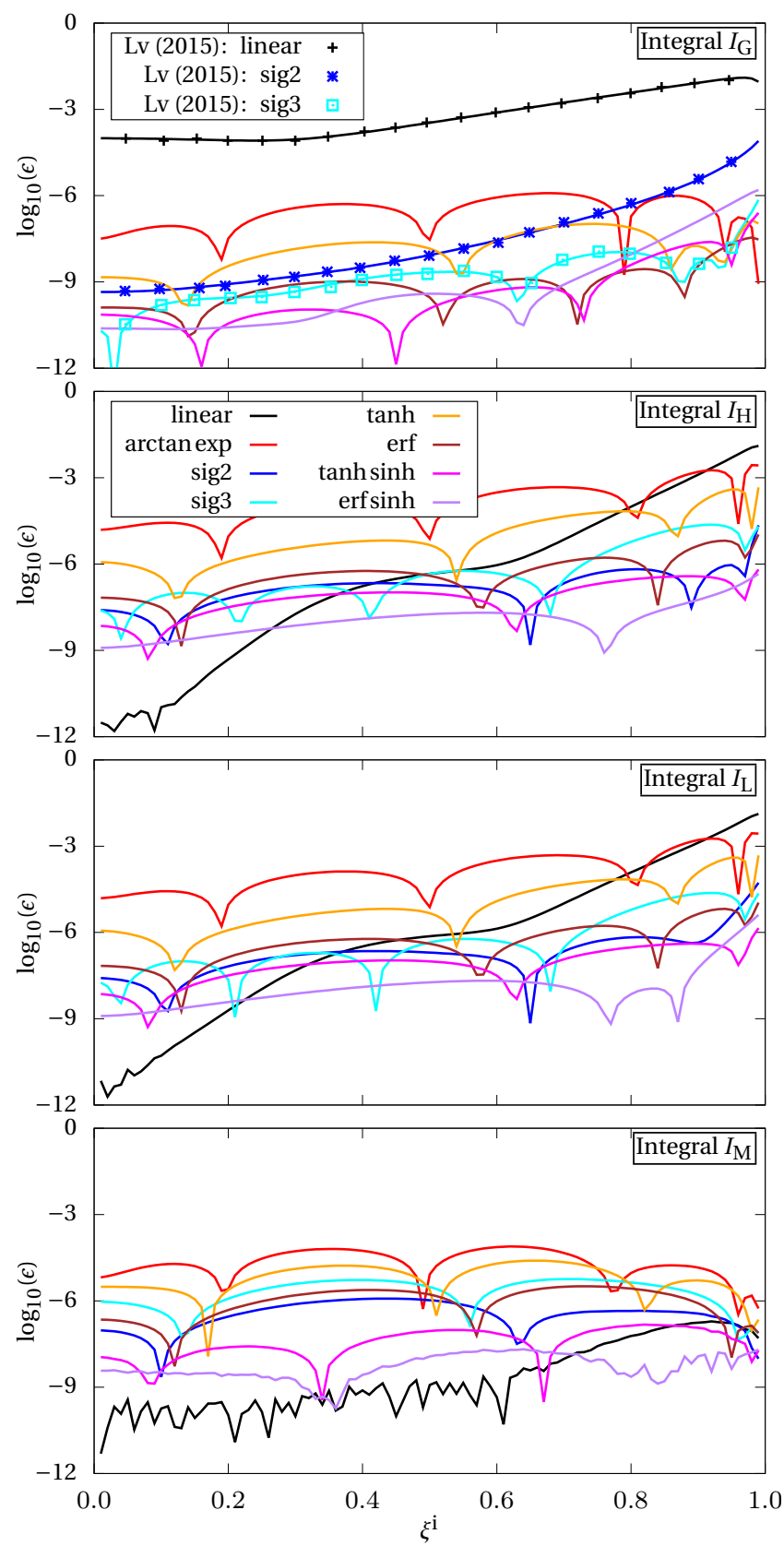

Figure 12: Relative error as the collocation point location moves along a line from $\xi^{\mathrm{i}}=(0,0)$ to $\xi^{\mathrm{i}}=(1,1)$ with $\xi_{1}^{\mathrm{i}}=\xi_{2}^{\mathrm{i}}=\xi^{\mathrm{i}}$. Number of integrations points for each triangular subdivision is $N_{\theta}=N_{\rho}=10$. results from Section 3 are clearly seen for $I_{\mathrm{G}}$. It is seen that particularly sig2 loses quite a bit of performance as the collocation point is nearer to the element vertex. This is also seen for sig3, erf sinh and tanh sinh to a lesser extent. For integrals $I_{\mathrm{H}}, I_{\mathrm{L}}$ and $I_{\mathrm{L}}$ results are very similar, showing a clear performance rank order where erf sinh is the best. The use of non-linear transformations is not crucial in these cases, although there is a pronounced performance lost as the collocation point is nearer the element vertex for the linear transformation, especially for $I_{\mathrm{H}}$ and $I_{\mathrm{L}}$. Therefore, in these cases non-linear transformations basically make relative error less dependent on the location of the collocation point.

Fig. 13 shows the convergence of the average relative errors $\varepsilon_{\text {avg }}$ as integration points $N=N_{\theta}=N_{\rho}$ for each triangular subdivision increase. The average relative error $\varepsilon_{\mathrm{avg}}$ is calculated from relative errors for the different locations of the collocation point, which is an indicator of the overall convergence of each non-linear transformation. Results show that non-linear transformations are indispensable for all integrals except for $I_{\mathrm{M}}$. For $I_{\mathrm{G}}$, erf has the best convergence rate, followed by tanh sinh and sig3 transformations. The worst in this case is the sig2 transformation. For the rest of integrals, the best non-linear transformation is erf sinh, followed by tanh sinh and sig2 transformations.

\section{Conclusions}

In this paper, it is proposed a numerical scheme for triangular and quadrilateral elements based on a conformal polar transformation and four novel non-linear transformations which completely removes all sources of angular quasi-singularities.

It is shown that there is no optimal non-linear transformation for all kernels. The most versatile solution is hence equipping the algorithm with many non-linear transformations in the arsenal, which are chosen depending on the integrand and some previous tests. In this sense, the four non-linear transformations proposed are the more robust and efficient since not only it has been demonstrated that they asymptotically remove these quasi-singularities but they also generally outperform sigmoidal transformations. From all, the tanh sinh transformation is consistently highly ranked in all the examples, and this should be the choice if only one should be selected.

\section{Acknowledgements}

The authors are grateful for the support from the Ministerio de Economía y Competitividad (MINECO) of Spain, the Agencia Estatal de Investigación (AEI) of Spain and FEDER through Research Projects BIA201457640-R and BIA2017-88770-R.

\section{A Other non-linear transformations for primary angular quasi-singularities}

arctan exp transformation Among other transformations, Khayat et al. [13] proposed the use of an arctan exp (or conversely ln tan) transformation. Taking into account that singularities are present at $\theta=\pi$ and $\theta=2 \pi$, for the present scheme it can be written as:

$$
\theta=\pi+2 \arctan (\exp (\tilde{\theta})), \quad \tilde{\theta}=\ln \left(\tan \left(\frac{\theta-\pi}{2}\right)\right)
$$

where $\lim _{\tilde{\theta} \rightarrow-\infty} \theta(\tilde{\theta})=\pi$ and $\lim _{\tilde{\theta} \rightarrow+\infty} \theta(\tilde{\theta})=2 \pi$. The Jacobian $J_{\mathrm{A} d}=$ $\partial \theta / \partial \tilde{\theta}$ is:

$$
J_{\mathrm{Ad}}=-\sin \theta=\frac{1}{\cosh \tilde{\theta}}
$$



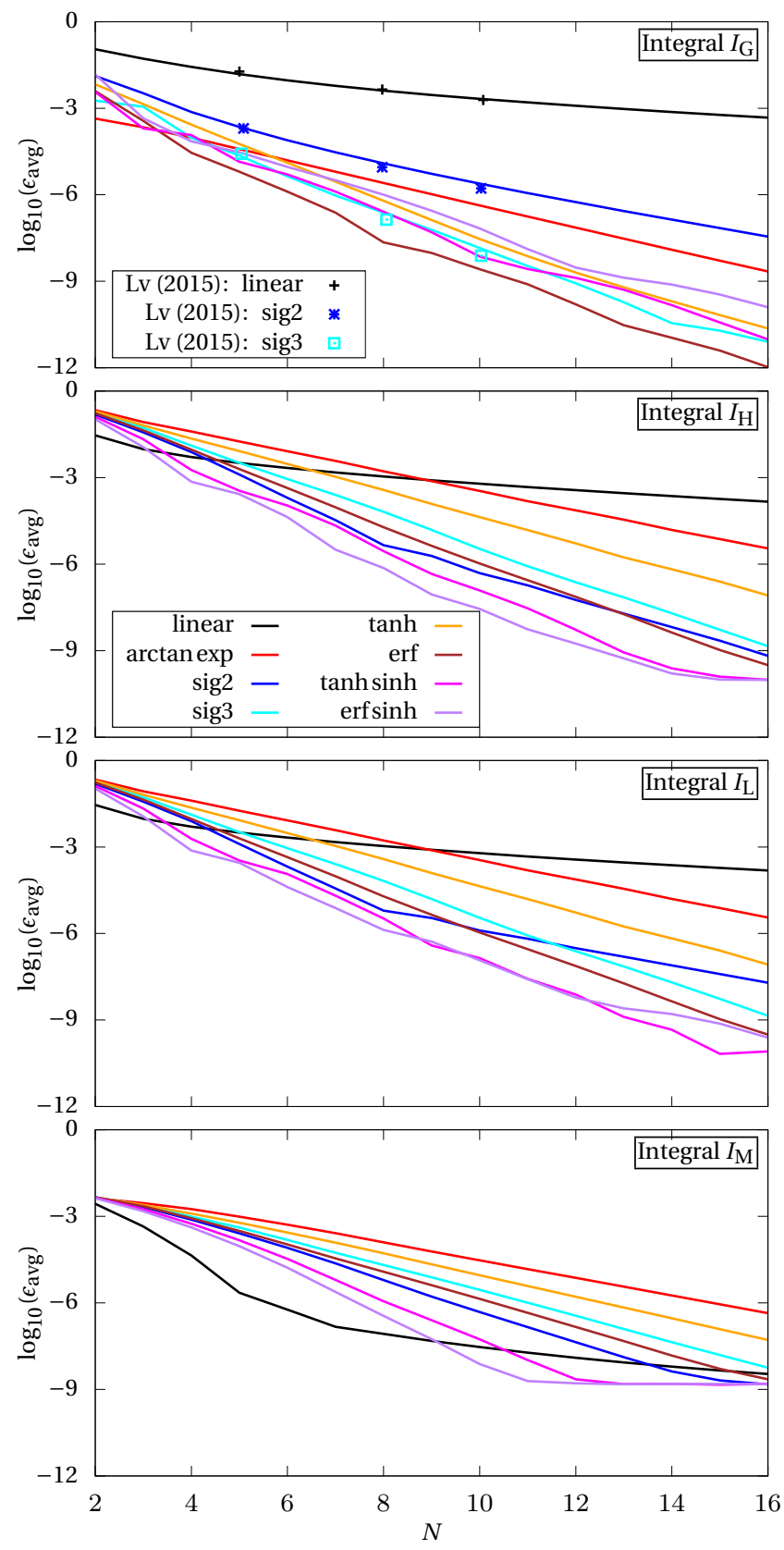

Figure 13: Convergence of average relative error $\varepsilon_{\text {avg }}$ when using $N=N_{\theta}=N_{\rho}$ integration points for each triangular subdivision and the domain $\tilde{\theta} \in\left[\tilde{\theta}^{(1)-S d}, \tilde{\theta}^{(2)-S d}\right]$ is given by:

$$
\tilde{\theta}^{(1)-\mathrm{S} d}=\ln \tan \frac{\theta^{(1)-\mathrm{S} d}-\pi}{2}, \tilde{\theta}^{(2)-\mathrm{S} d}=\ln \tan \frac{\theta^{(2)-\mathrm{S} d}-\pi}{2}
$$

Therefore, it maps $\tilde{\boldsymbol{\theta}} \in\left[\tilde{\boldsymbol{\theta}}^{(1)-S d}, \tilde{\boldsymbol{\theta}}^{(2)-S d}\right]$ onto $\theta \in[\pi, 2 \pi]$. This transformation can also be written in terms of the Gudermannian function, and also in a number of combinations between trigonometric and hyperbolic functions and its inverses due to their relationships. Khayat's arctan exp transformation completely removes the strong quasi-singularities since its Jacobian (Eq. (44) cancels it out perfectly $\left(J_{\mathrm{A} d} \cdot J_{\mathrm{UAd}}\right.$ is a constant).

Any integrand from static parts of fundamental solutions can ultimately be written in terms of elementary trigonometric functions $\sin \theta$ and $\cos \theta$. Unlike other transformations, this transformation transform trigonometric functions into simple hyperbolic functions:

$$
\sin \theta=-\frac{1}{\cosh \tilde{\theta}}, \cos \theta=\tanh \tilde{\theta}
$$

which may facilitate future numerical analyses of this transformation.

Sigmoidal transformations of order $m$ Rong et al. [19] proposed the use of sigmoidal transformations taken from [11]. These sigmoidal transformations are rational functions which allow the concentration of integration points at the end-points of the interval. For the present scheme, the sigmoidal transformation of order $m$ can be written as:

$$
\theta=\pi+\pi \frac{\left(\frac{\tilde{\theta}+1}{2}\right)^{m}}{\left(\frac{\tilde{\theta}+1}{2}\right)^{m}+\left(1-\frac{\tilde{\theta}+1}{2}\right)^{m}}
$$

which maps $\tilde{\theta} \in[-1,1]$ onto $\theta \in[\pi, 2 \pi]$. The actual integration limits $\tilde{\theta}^{(1)-S d}$ and $\tilde{\theta}^{(2)-S d}$ are found by inverting Eq. 47) and substituting $\theta$ by $\theta^{(1)-S d}$ and $\theta^{(2)-S d}$ respectively. For the case $m=2, \tilde{\theta}(\theta)$ can be written in a very simple form:

$$
\tilde{\theta}=\frac{\pi / 2-\sqrt{(\theta-\pi)(2 \pi-\theta)}}{\theta-3 \pi / 2}
$$

The Jacobian $J_{\mathrm{A} d}=\partial \theta / \partial \tilde{\theta}$ in this case becomes:

$$
J_{\mathrm{A} d}=\pi \frac{(1-\tilde{\theta})(1+\tilde{\theta})}{\left(1+\tilde{\theta}^{2}\right)^{2}}
$$

For the case $m=3, \tilde{\theta}(\theta)$ is a lengthy expression, but the Jacobian can be written as:

$$
J_{\mathrm{A} d}=\frac{3 \pi}{2} \frac{(1-\tilde{\theta})^{2}(1+\tilde{\theta})^{2}}{\left(1+3 \tilde{\theta}^{2}\right)^{2}}
$$

\section{References}

[1] J. D. R. Bordón, J. J. Aznárez, and O. Maeso. Dynamic model of open shell structures buried in poroelastic soils. Computational Mechanics, 60(2):269-288, 2017. doi:10.1007/ s00466-017-1406-3

[2] C. A. Brebbia. The birth of the boundary element method from conception to application. Engineering Analysis with Boundary Elements, 77:iii-x, 2017. doi:10.1016/j.enganabound.2016. 12.001

[3] A. H. D. Cheng and D. T. Cheng. Heritage and early history of the boundary element method. Engineering Analysis with Boundary Elements, 29:268-302, 2005. doi:10.1016/j.enganabound. 2004.12.001 
[4] T. A. Cruse. Numerical solutions in three dimensional elastostatics. International Journal of Solids and Structures, 5:1259-1274, 1969. doi:10.1016/0020-7683(69)90071-7.

[5] T. A. Cruse. Boundary Integral Equation - A Personal View. Electronic Journal of Boundary Elements, 1(1):19-25, 2003. doi: 10.14713/ejbe.v1i1.745

[6] J. Domínguez, M. P. Ariza, and R. Gallego. Flux and traction boundary elements without hypersingular or strongly singular integrals. International Journal for Numerical Methods in Engineering, 48:111-135, 2000. doi:10.1002/(SICI) 1097-0207(20000510) 48:1<111: : AID-NME870>3.0.CO;2-Y

[7] A. Frangi and M. Guiggiani. A direct approach for boundary integral equations with high-order singularities. International Journal for Numerical Methods in Engineering, 49:871898, 2000. doi:10.1002/1097-0207(20001110)49:7<871:: AID-NME979>3.0.CO;2-V

[8] M. Guiggiani and A. Gigante. A general algorithm for muldimensional cauchy principal value integrals in the boundary element method. Journal of Applied Mechanics, Transactions ASME, 57:906-915, 1990. doi:10.1115/1.2897660

[9] M. Guiggiani, G. Krishnasamy, T.J. Rudolphi, and F.J. Rizzo. A general algorithm for the numerical solution of hypersingular boundary integral equations. Journal of Applied Mechanics, Transactions ASME, 59:604-614, 1992. doi : 10.1115/1.2893766

[10] K. Hayami. A Projection Transformation Method for Nearly Singular Surface Boundary Element Integrals, volume 73 of Lecture Notes in Engineering. Springer-Verlag, 1992. doi:10.1007/ 978-3-642-84698-4

[11] P. R. Johnston. Application of sigmoidal transformations to weakly singular and near-singular boundary element integrals. International Journal for Numerical Methods in Engineering, 45:13331348, 1999. doi:10.1002/(SICI) 1097-0207(19990810)45: 10<1333: :AID-NME632>3.0.CO;2-Q

[12] G. Karami and D. Derakhshan. An efficient method to evaluate hypersingular and supersingular integrals in boundary integral equations analysis. Engineering Analysis with Boundary Elements, 23:317-326, 1999. doi:10.1016/S0955-7997(98)00085-X.

[13] M. A. Khayat and D. R. Wilton. An improved transformation and optimized sampling scheme for the numerical evaluation of singular and near-singular potentials. IEEE Antennas and Wireless Propagation Letters, 7:377-380, 2008. doi:10.1109/LAWP. 2008. 928461

[14] H. B. Li, G. M. Han, and H. A. Mang. A new method for evaluating singular integrals in stress analysis of solid by the boundary element method. International Journal for Numerical Methods in Engineering, 33:2071-2098, 1985. doi:10.1002/nme. 1620211109

[15] J.-H Lv, X.-T Feng, F. Yan, and Q. Jiang. Efficient evaluation of integrals with kernel $1 / r^{\chi}$ for quadrilateral elements with irregular shape. Engineering Analysis with Boundary Elements, 61:33-41, 2015. doi:10.1016/j.enganabound.2015.06.010

[16] H. Ma and N. Kamiya. Distance transformation for the numerical evaluation of near singular boundary integrals with various kernels in boundary element method. Engineering Analysis with Boundary Elements, 26:329-339, 2002. doi:10.1016/S0955-7997(02) 00004-8
[17] M. Mori and M. Sugihara. The double-exponential transformation in numerical analysis. Journal of Computational and Applied Mathematics, 127:287-296, 2001. doi:10.1016/ S0377-0427(00) 00501-X

[18] P. Partheymüler, R. A. Bialecki, and G. Kuhn. Self-adapting algorithm for evaluation of weakly singular integrals arising in the boundary element method. Engineering Analysis with Boundary Elements, 14:285-292, 1994. doi:10.1016/0955-7997(94) 90044-2.

[19] J. Rong, L. Wen, and J. Xiao. Efficiency improvement of the polar coordinate transformation for evaluating bem singular integrals on curved elements. Engineering Analysis with Boundary Elements, 38:83-93, 2014. doi:10.1016/j.enganabound.2013.10.014.

[20] M. Tanaka, V. Sladek, and J. Sladek. Regularization techniques applied toboundary element methods. Applied Mechanics Reviews, 47:457-499, 1994. doi:10.1115/1.3111062 